\title{
Acoustically modulated optical emission of hexagonal boron nitride layers
}

Cite as: Appl. Phys. Lett. 114, 171104 (2019); https://doi.org/10.1063/1.5093299

Submitted: 19 February 2019 . Accepted: 17 April 2019 . Published Online: 02 May 2019

Fernando likawa (D), Alberto Hernández-Mínguez (D), Igor Aharonovich (D), Siamak Nakhaie, Yi-Ting Liou (D), João Marcelo J. Lopes, and Paulo V. Santos (i)

\section{COLLECTIONS}

This paper was selected as Featured
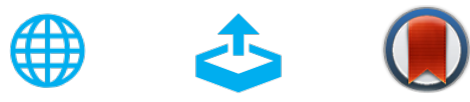

View Online

Export Citation

\section{ARTICLES YOU MAY BE INTERESTED IN}

A fiber-integrated nanobeam single photon source emitting at telecom wavelengths

Applied Physics Letters 114, 171101 (2019); https://doi.org/10.1063/1.5089907

High performance of AlGaN deep-ultraviolet light emitting diodes due to improved vertical carrier transport by delta-accelerating quantum barriers

Applied Physics Letters 114, 172105 (2019); https://doi.org/10.1063/1.5093160

Improved carrier doping strategy of monolayer $\mathrm{MoS}_{2}$ through two-dimensional solid electrolyte of $\mathrm{YBr}_{3}$

Applied Physics Letters 114, 171601 (2019); https://doi.org/10.1063/1.5093712

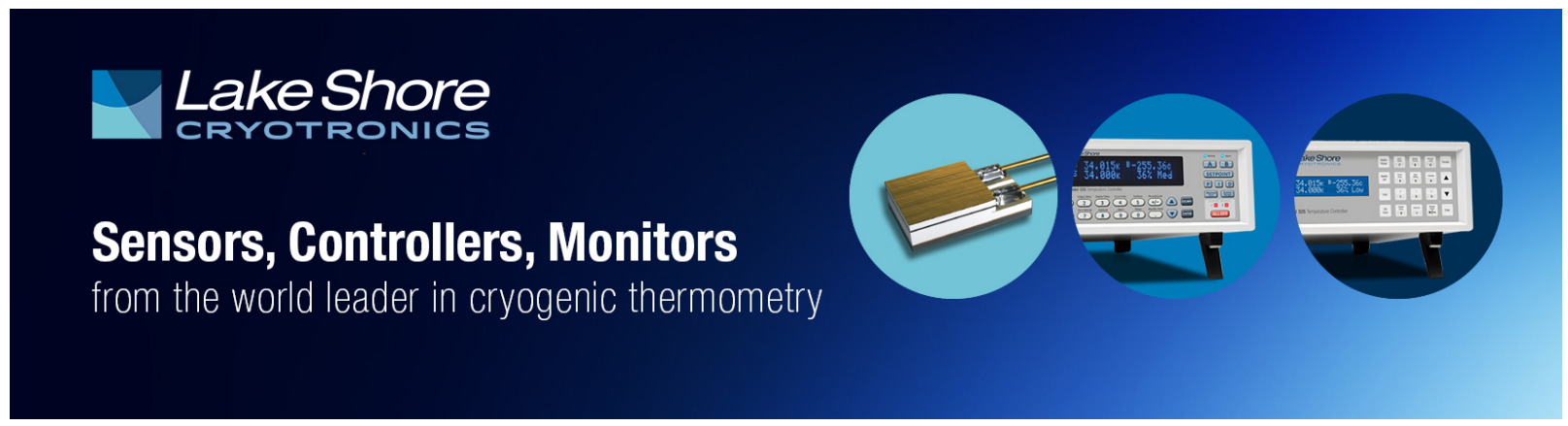




\title{
Acoustically modulated optical emission of hexagonal boron nitride layers 8
}

\author{
Cite as: Appl. Phys. Lett. 114, 171104 (2019); doi: 10.1063/1.5093299 \\ Submitted: 19 February 2019 - Accepted: 17 April 2019 . \\ Published Online: 2 May 2019
}

Fernando likawa, ${ }^{1,2}$ (D) Alberto Hernández-Mínguez, ${ }^{1, a)}$ (D) Igor Aharonovich, ${ }^{3}$ (D) Siamak Nakhaie, ${ }^{1}$ Yi-Ting Liou, João Marcelo J. Lopes, and Paulo V. Santos ${ }^{\top}$

\author{
AFFILIATIONS \\ ${ }^{7}$ Paul-Drude-Institut für Festkörperelektronik, Leibniz-Institut im Forschungsverbund Berlin e.V., Hausvogteiplatz 5-7, 10117 Berlin, \\ Germany \\ ${ }^{2}$ Institute of Physics, State University of Campinas, 13083-859 Campinas-SP, Brazil \\ ${ }^{3}$ School of Mathematical and Physical Sciences, University of Technology Sydney, Ultimo, New South Wales 2007, Australia \\ a)alberto.h.minguez@pdi-berlin.de
}

\begin{abstract}
We investigate the effect of surface acoustic waves on the atomic-like optical emission from defect centers in hexagonal boron nitride layers deposited on the surface of a $\mathrm{LiNbO}_{3}$ substrate. The dynamic strain field of the surface acoustic waves modulates the emission lines resulting in intensity variations as large as $50 \%$ and oscillations of the emission energy with an amplitude of almost 1 meV. From a systematic study of the dependence of the modulation on the acoustic wave power, we determine a hydrostatic deformation potential of about 40 meV/\% for defect centers in this two-dimensional material. Furthermore, we show that the dynamic piezoelectric field of the acoustic wave could contribute to the stabilization of the optical properties of these emission centers. Our results show that surface acoustic waves are a powerful tool to modulate and control the electronic states of two-dimensional materials.
\end{abstract}

Published under license by AIP Publishing. https://doi.org/10.1063/1.5093299

Defect centers in solids have attracted much attention recently due to their atomic-like optical emission characterized by strong and sharp lines. Typical examples are the nitrogen-vacancy defects in diamond ${ }^{1,2}$ and defect centers in SiC. ${ }^{3}$ These centers act as single photon sources, thus becoming promising candidates for application in quantum information processing. One of the challenges related to these luminescent centers in solids is to find mechanisms for the efficient control of their optoelectronic properties. To this end, surface acoustic waves (SAWs) are an interesting approach because their strain and piezoelectric fields oscillating with frequencies in the range of hundreds of $\mathrm{MHz}$ can couple efficiently to defect centers placed close to the surface of the vibrating substrate. ${ }^{4-6}$

In this manuscript, we discuss the dynamic modulation of optically active centers in hexagonal boron nitride (h-BN) by SAWs. h-BN is a two-dimensional (2D) crystal with a graphene-like honeycomb atomic lattice. In contrast to graphene, h-BN displays a wide energy bandgap $(\sim 6 \mathrm{eV})$, which makes it an exceptional insulator in, e.g., van der Waals heterostructures. ${ }^{7,8}$ It has recently been demonstrated that h-BN can host defect centers acting as single photon emitters in both the visible ${ }^{9-21}$ and ultraviolet spectral ranges, ${ }^{22}$ making this material promising for quantum optics. To this end, it is necessary to develop techniques for the tuning of their emission energies. This can be achieved by applying either static strain ${ }^{23,24}$ or electric fields. ${ }^{25-28}$ Here, we demonstrate that SAWs can couple to defect centers contained in two kinds of h-BN samples, namely, multi-layer-thick flakes and few-layer-thick films.

The samples containing multilayer h-BN flakes with a thickness of about $200 \mathrm{~nm}$ and a lateral size of about $1 \mu \mathrm{m}$ (obtained from Graphene Supermarket) were prepared by drop-casting the flakes on a $127^{\circ} \mathrm{Y}$-cut $\mathrm{LiNbO}_{3}$ substrate followed by an annealing step at $850^{\circ} \mathrm{C}$ for $30 \mathrm{~min}$. in an Argon atmosphere (1 Torr) to activate their emission properties. ${ }^{9,10}$ Finally, SAW delay lines consisting of pairs of interdigital transducers (IDTs) were patterned on $\mathrm{LiNbO}_{3}$ by optical lithography and lift-off metallization, cf. Fig. 1(a). In contrast, few-layer-thick h-BN films (about $1 \mathrm{~nm}$ thick) were grown on nickel by molecular beam epitaxy (MBE). ${ }^{29}$ Then, an area of about $7 \times 6 \mathrm{~mm}^{2}$ was transferred onto $\mathrm{LiNbO}_{3}$ using a wet transfer technique. ${ }^{30}$ In this case, $\mathrm{LiNbO}_{3}$ was patterned with acoustic delay lines before transferring the h-BN film. In addition, we did not perform any post-transfer annealing in these samples. This allowed us to directly compare their optical performance with previous results on $\mathrm{SiO}_{2} / \mathrm{Si}$, where the MBE-grown films were also not annealed. ${ }^{30}$ 
(a)

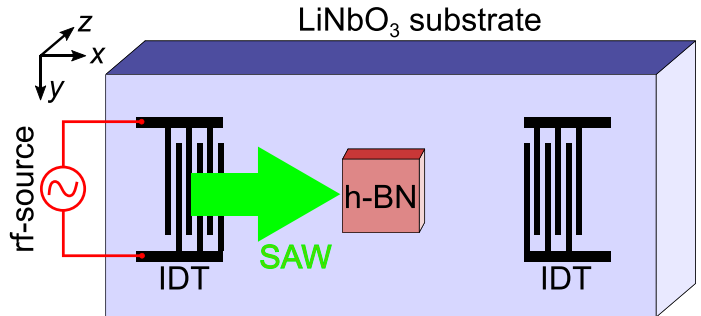

(b)

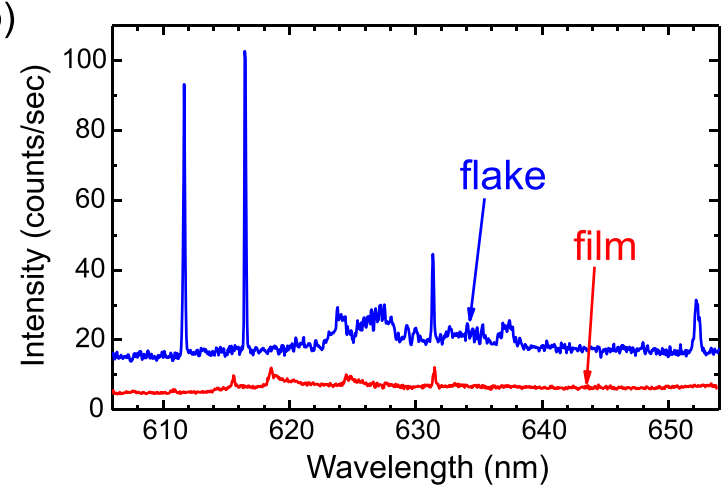

FIG. 1. (a) Schematic diagram of the samples. They contain two interdigital transducers (IDTs) patterned at the surface of $\mathrm{LiNbO}_{3}$. An rf signal applied to one of the IDTs excites a SAW propagating along the region where the hexagonal boron nitride ( $h-B N)$ is deposited. (b) Low temperature $(5 K)$ luminescence spectrum of defect centers in a multilayer-thick h-BN flake (blue curve) and in a few-layer-thick film (red curve).

The samples were investigated in a He cryostat adapted for micro-photoluminescence ( $\mu$-PL) measurements with a spatial resolution of about one micrometer (Attocube Confocal Microscope). The experiments were performed at a nominal temperature of $5 \mathrm{~K}$. The defect centers were optically excited by a $532 \mathrm{~nm}$ solid-state laser beam focused onto the sample using an objective with a large numerical aperture of about 0.8 . The emitted light was collected by the same objective, coupled into a single-mode optical fiber, and sent into a $0.5 \mathrm{~m}$-long monochromator equipped with a $900 \mathrm{~mm}^{-1}$ grating and a Si-based charge-coupled device (CCD) camera. The SAWs were excited by applying an $\mathrm{rf}$ signal of appropriate frequency to one of the IDTs using an rf generator connected to an amplifier (about $23 \mathrm{~dB}$ amplification).

To detect SAW-induced changes in the PL spectra with the required large signal/noise ratio, we used the modulation method described in Ref. 31 . Here both the rf source and the excitation laser are amplitude-modulated with a modulation frequency of about $300 \mathrm{~Hz}$. The spectra are recorded using SAW and laser excitation modulated out of phase, i.e., the sample is exposed to the laser when the rf signal is switched off (SAW-OFF spectrum, $I_{\text {off }}$ ), as well as in phase under laser excitation of the sample when the rf signal is switched on (SAW-ON spectrum, $\left.I_{o n}\right)$. We repeat accumulation sequences SAW-OFF - SAW-ON - SAW-ON - SAW-OFF over typically 20 times and average the corresponding ON and OFF measurements (see supplementary material). This acquisition method increases the signal/noise ratio due to the long accumulation times and, most important, minimizes the effects of systematic temperature and laser power fluctuations, as well as those related to spectral wandering typically observed in $\mathrm{h}-\mathrm{BN}$ emission centers. ${ }^{1.5}$
The blue curve in Fig. 1(b) shows a typical PL spectrum of a hBN flake, which consists of several sharp lines distributed along the visible spectral range (between 550 and $800 \mathrm{~nm}$ ). ${ }^{11,12}$ We observed similar sharp lines in the transferred h-BN films, cf. red curve in Fig. 1(b), but with a much weaker emission intensity. There are several possible reasons for this weaker emission. First, the flakes were annealed to activate luminescent defects, while the films did not undergo such a process. Second, the flakes contain much more layers than the film. This means that, in principle, the number of defects which are excited by the laser spot and emit light at a certain wavelength should be larger in the flakes than in the film. However, light centers in h-BN show typically a large spectral distribution. ${ }^{11,15}$ Therefore, the probability of finding two or more centers emitting at exactly the same wavelength is relatively low. Finally, the interaction of the defect centers with the $\mathrm{LiNbO}_{3}$ substrate may also play a role. Actually, the intensity of the emission lines in the MBE film was weaker than the ones measured under comparable experimental conditions in similar samples transferred to $\mathrm{SiO}_{2} / \mathrm{Si}^{30}$ It has been reported that electric-field-induced charging effects can tune the brightness of luminescent centers. ${ }^{25,32,33} \mathrm{As}^{\mathrm{LiNbO}} 3$ is a ferroelectric material, its spontaneous polarization fields could weaken the luminescence intensity of h-BN defects close to its surface. This effect is expected to affect the thin films more significantly than the flakes since the thicker flakes also contain defects at a larger distance from $\mathrm{LiNbO}_{3}$, which are therefore optically brighter.

Figure 2 displays the PL spectra of one of the emission lines of an h-BN flake, recorded in the absence (Fig. 2(a), SAW OFF) and in the presence of SAW excitation (Fig. 2(b), SAW ON). The measurements were performed for several frequencies of the rf signal applied to the IDT. As the $I_{\text {off }}$ spectra are recorded with the exciting laser and rf signal out of phase, we do not expect any effect of the SAW on the emission properties. We have fitted the $\mathrm{I}_{\text {off }}$ spectra by the following Lorentzian function [black curves in Fig. 2(a)]:

$$
I_{\text {off }}(\lambda)=I_{0}+\frac{2 A}{\pi} \frac{w}{4\left(\lambda-\lambda_{c}\right)^{2}+w^{2}},
$$

where $A, w$, and $\lambda_{c}$ are the area, width, and center of the emission line, respectively, and $I_{0}$ accounts for the background signal. While the width remains quite stable at an average value of $0.28 \pm 0.03 \mathrm{~nm}$, the peak center shifts from $630.4 \mathrm{~nm}$ to $630.7 \mathrm{~nm}$ during the experiment. Spectra measured as a function of time (see supplementary material) indicate that this shift is a consequence of spectral and intensity fluctuations (spectral diffusion) probably caused by aleatory changes in the ionization state of nearby charge traps like shallow impurities or defects. ${ }^{15,34-37}$ These fluctuations are also responsible for the relatively broad emission line and for the partial deviation of the line shape from that of a single peak.

The corresponding $I_{o n}$ spectra, in contrast, show an additional line broadening for a certain range of $\mathrm{rf}$ frequencies, accompanied by a decrease in the amplitude. We interpret this change in the shape of the line as caused by the dynamic strain field of the SAW: The oscillating tensile and compressive strains modulate periodically the crystal structure of the luminescent center, thus leading to the oscillation of its optical transition energy around the equilibrium value. ${ }^{23,24}$ As the acquisition time of the $\mu$-PL spectra is much larger than the SAW period, the energy oscillation manifests as a broadening of the emission line and a reduction of its maximum intensity, as shown in Fig. 2(b). 

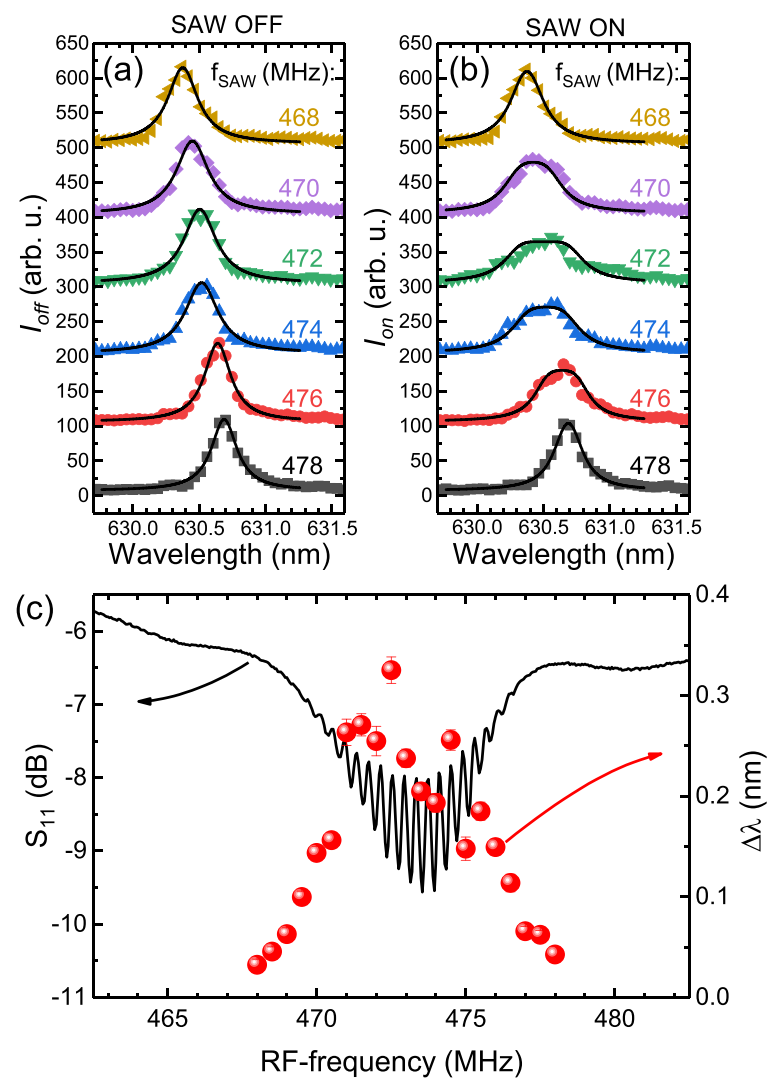

FIG. 2. (a) $I_{\text {off }}$ and (b) $I_{\text {on }}$ spectra of a luminescent center as a function of the if frequency applied to the IDT that launches the SAW, $f_{S A W}$ (nominal if power of $-1 \mathrm{dBm}$ ). The black curves are fittings to the data according to Eqs. (1) and (2). The data are vertically shifted for clarity. (c) rf-power reflection coefficient $S_{11}$ of the IDT (black curve) and amplitude of the spectral oscillation, $\Delta \lambda$ (red dots), calculated for several if frequencies across the resonance of the IDT.

Similar broadening effects under dynamic strain have been commonly observed in the luminescence spectra of quantum dots, ${ }^{38-41}$ as well as in the electronic spin resonance of defects in diamond, ${ }^{42}$ and in the Raman lines of semiconductors. ${ }^{31}$

To quantify the strength of the optomechanical coupling, we have fitted the $I_{\text {on }}$ spectra of Fig. 2(b) according to the formula ${ }^{39,42-44}$

$I_{\text {on }}(\lambda)=I_{0}+f_{\text {SAW }} \int_{0}^{1 / f_{\text {SAW }}} \frac{2 A}{\pi} \frac{w}{4\left\{\lambda-\left[\lambda_{c}+\Delta \lambda \cos \left(2 \pi f_{S A W} t\right)\right]\right\}^{2}+w^{2}} d t$.

Here, the term in the integrand is the Lorentzian function with the values of $A, w$, and $\lambda_{c}$ obtained from the fitting of the corresponding $I_{\text {off }}$ spectra in Fig. 2(a), and $f_{S A W}$ is the frequency of the rf signal that launches the SAW. The amplitude of the SAW-induced spectral oscillation is given by $\Delta \lambda$, which is the only free parameter in the fitting. We display in Fig. 2(c) the dependence of $\Delta \lambda$ on the rf frequency (red dots) and compare it with the rf-power reflection coefficient, $S_{11}$, of the IDT (black curve, measured using a vector network analyzer). Non-vanishing values of $\Delta \lambda$ are only observed for $\mathrm{rf}$ frequencies within the minimum in the $S_{11}$ spectrum, corresponding to the frequency range for efficient SAW excitation by the IDT. The maximum intensity suppression (of about 50\%) and broadening of the PL emission occur for SAW frequencies around $473 \mathrm{MHz}$ and are well reproduced by our fitting supposing a value of $\Delta \lambda$ up to $0.3 \mathrm{~nm}$. This corresponds to SAW-induced oscillations of the emission energy with amplitudes of almost $1 \mathrm{meV}$.

As $\mathrm{LiNbO}_{3}$ is a strong piezoelectric material, the SAW strain field is accompanied by an oscillating electric field that could also, in principle, be responsible for the modulation of the emission line. It has recently been reported that electric fields of $1 \mathrm{GV} / \mathrm{m}$ can induce Stark shifts in h-BN defect centers on the order of $10 \mathrm{~nm} .^{25,26,28}$ However, the SAW-induced piezoelectric fields in $\mathrm{LiNbO}_{3}$ are typically on the order of $1 \mathrm{MV} / \mathrm{m}$, which means that the expected Stark shifts are about $0.01 \mathrm{~nm}$. Therefore, we can neglect the contribution of the SAW piezoelectric fields to the observed modulation.

Another evidence supporting the preeminent role of the strain over the piezoelectric field in the acoustic modulation is the fact that, out of twenty studied emission centers, only three effectively underwent the acoustic modulation. This indicates that the quality of the physical contact between the flakes and the $\mathrm{LiNbO}_{3}$ substrate is critical for the coupling of the SAW into the h-BN. In addition, the amount of strain that can be transmitted from the bottom to the top of the multilayer flakes can be strongly limited due to the weak Van-der-Waals interaction between adjacent monolayers in $2 \mathrm{D}$ materials. ${ }^{23,45}$ As a matter of fact, the emission centers undergoing acoustic modulation were optically weaker than the unperturbed ones, thus supporting our assumption that the $\mathrm{LiNbO}_{3}$ substrate affects the optical properties of emission centers in close contact to the substrate.

Although all three centers undergoing acoustic modulation showed similar behavior, only the one discussed in Fig. 2 was stable enough to perform a systematic study of the SAW-induced effects on the emission line. Figures 3(a) and 3(b) show the dependence of the $I_{\text {off }}$ and $I_{o n}$ spectra on the nominal rf power applied by the rf generator. As expected, the $I_{\text {off }}$ spectra remain unperturbed by the SAW, while the SAW-induced broadening of the $I_{o n}$ spectra increases with the amplitude of the rf power. Figure 3(c) displays the fitted values of $\Delta \lambda$ as a function of the amplitude of the hydrostatic strain, $\varepsilon_{0}=\varepsilon_{x x}+\varepsilon_{z z}$, at the surface of $\mathrm{LiNbO}_{3}$ for the different nominal rf powers (since the SAW is a Rayleigh mode, strain is generated along the $x$ and $z$ directions, but not along the $y$ direction $^{46}$ ). To determine the values of $\varepsilon_{0}$, we first calculated the SAW power density from the nominal rf power applied to the IDT and the reflection coefficient of Fig. 2(c). We then related the SAW power density to $\varepsilon_{0}$ by solving numerically the coupled elastic and electromagnetic equations for the $\mathrm{LiNbO}_{3}$ substrate. As expected, $\Delta \lambda$ increases linearly with $\varepsilon_{0}$ at a rate of $12.9 \pm 0.2 \mathrm{~nm}$ per $\%$ of strain, which corresponds to a deformation potential of about $40 \mathrm{meV} / \%$. This value is ten times larger than the ones reported for multilayer h-BN under static strain, ${ }^{23,24}$ but similar to the result recently obtained for SAW-modulated defects in h-BN powder. ${ }^{47}$

Finally, we discuss the MBE-grown h-BN films. As mentioned before, the emission lines of these samples are surprisingly weak. In addition, they undergo significant spectral diffusion. These features masked the observation of the acoustic broadening reported above for the flakes. Under the application of the SAW, however, we observed a stabilization of the optical emission for some light centers. As an example, Fig. 4(a) shows a series of PL spectra recorded as a function of time (time delay of $20 \mathrm{~s}$ between two successive acquisitions) for a 

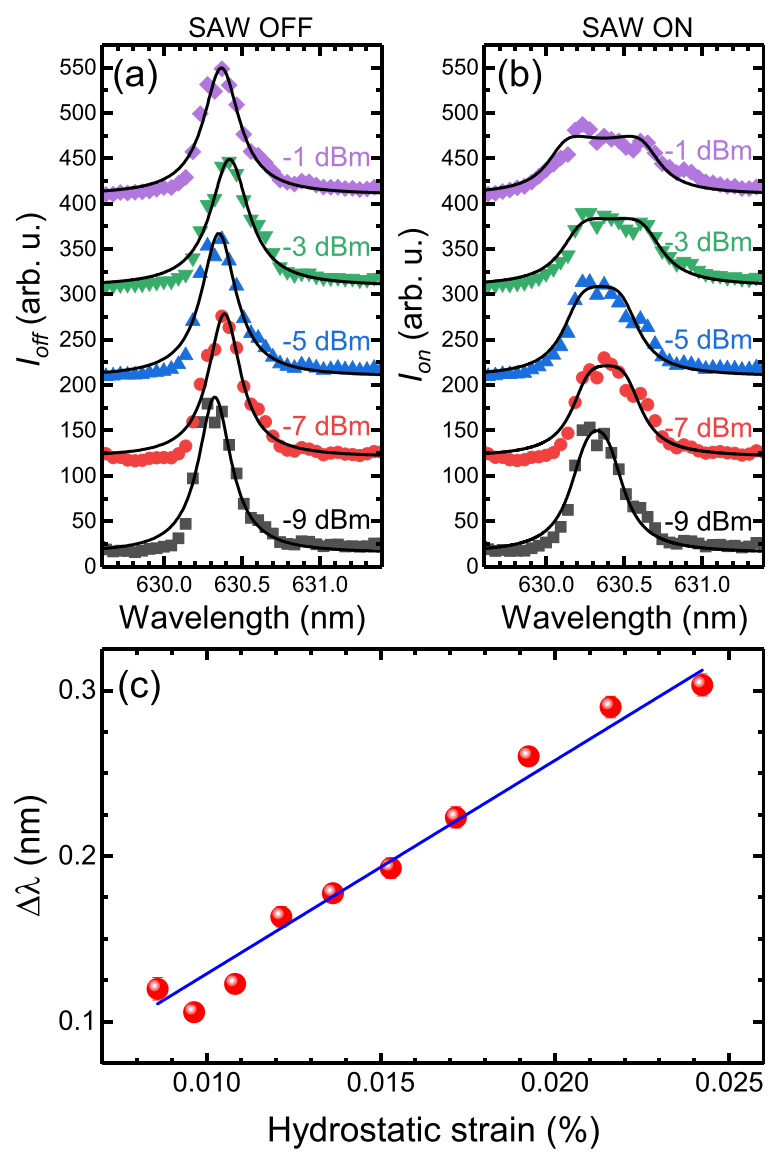

FIG. 3. (a) $I_{\text {off }}$ and (b) $I_{\text {on }}$ spectra as a function of nominal rf-power (recorded for $f_{S A W}=473.3 \mathrm{MHz}$ ). The black curves are fits according to Eqs. (1) and (2). The data are vertically shifted for clarity. (c) Amplitude of the spectral oscillation, $\Delta \lambda$, with respect to the amplitude of the SAW-induced hydrostatic strain at the surface of $\mathrm{LiNbO}_{3}$. The blue line is a linear fit to the data.

sharp peak emitting at $624 \mathrm{~nm}$ in the absence of SAWs. The emission wavelength fluctuates in time between two well-defined values at 623.5 and $624.1 \mathrm{~nm}$. The intensity of the emission line also fluctuates even though laser excitation density and sample temperature were kept constant during the experiment. Figure 4(b) shows the same sequence of spectra in the presence of a SAW of $f_{S A W}=513.50 \mathrm{MHz}$ and a nominal rf power of $0 \mathrm{dBm}$ (the IDTs in this sample were designed to launch SAWs with a different frequency from the ones for the flakes). Under these experimental conditions, the $623.5 \mathrm{~nm}$ peak disappeared. This effect was systematically observed in three out of ten luminescent centers studied here, and it was reproducible every time that we switched on the acoustic waves.

Following the model discussed before for the flakes, the spectral fluctuations in the absence of SAWs suggest a strong coupling of the luminescent center to the ionization state of a nearby shallow charge trap. $^{37,48}$ Therefore, we interpret the stabilization of the luminescence as caused by the interaction of the SAW with the nearby charge trap. It is known that the piezoelectric fields of SAWs can control the charge population of shallow quantum-dot-like centers by dynamically
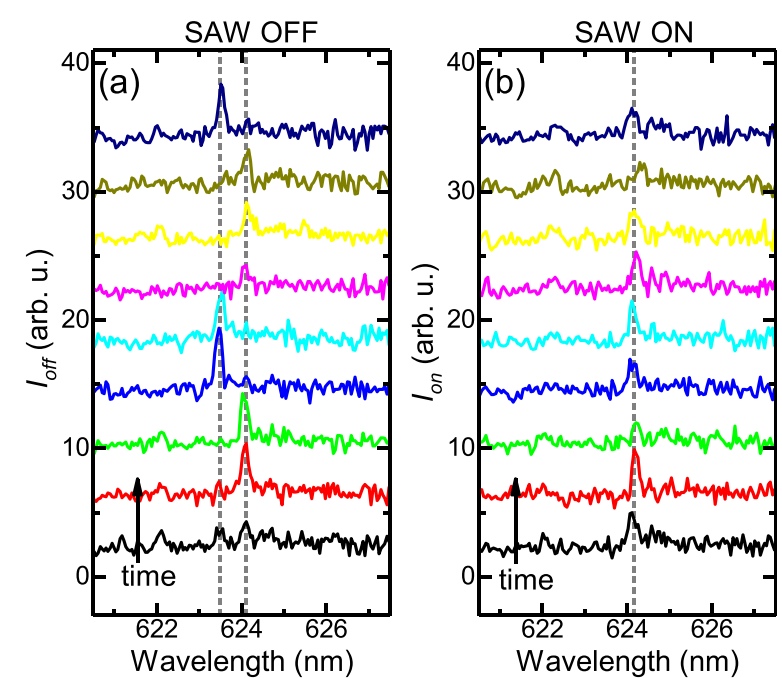

FIG. 4. Time evolution of the light emitted by a center in the MBE-grown film (a) in the absence of SAW and (b) when the SAW is applied. The data are vertically shifted for clarity. The spectra were recorded sequentially starting from the bottom (integration time of $20 \mathrm{~s}$ per spectrum). The vertical dashed lines mark the average positions of the peaks.

injecting and/or extracting charge carriers. ${ }^{41,49-52}$ According to this, we attribute the quenching of the $623.5 \mathrm{~nm}$ peak in Fig. 4(b) to the fact that the dynamic SAW piezoelectric field favors a certain ionization state of the nearby shallow trap. This can occur either by continuously injecting charge carriers into the trap or by extracting the charge carriers as soon as they are trapped. Supposing that the high and low energy peaks in Fig. 4(a) reflect the interaction of the light center with a nearby shallow trap in its negative and neutral charge states, respectively, then the suppression of the high energy peak in Fig. 4(b) would indicate that charge carrier extraction is the dominant mechanism in this case. Although a more comprehensive understanding of this mechanism requires additional studies that go beyond the scope of this manuscript, this result suggests that SAWs could be a helpful tool to reduce the energy fluctuation of the emission lines in the case of unstable emitters, as in our MBE-grown h-BN film.

In conclusion, we have investigated the interaction of SAWs with optically active defect centers in h-BN flakes and films transferred to the surface of $\mathrm{LiNbO}_{3}$. In the case of the flakes, we have demonstrated the modulation of the emission lines by the alternating acoustic field and estimated a deformation potential for the defects of about $40 \mathrm{meV} / \%$. For the MBE-grown film, although the weakness of the emission lines and their strong spectral diffusion prevented us from observing the acoustically induced broadening, the presence of the SAW fields suppressed the spectral fluctuations, thus leading to a more stable optical emission of the centers.

See supplementary material for luminescence spectra as a function of time for a light center in h-BN flakes.

The authors would like to thank Dr. Snežana Lazić for discussions and Dr. Lutz Schrottke for a critical reading of this manuscript. F.I. and I.A. acknowledge the Alexander von Humboldt Foundation for financial support. F.I. acknowledges 
Conselho Nacional de Desenvolvimento Científico e Tecnológico (Nos. 305769/2015-4 and 432882/2018-9) for financial support.

\section{REFERENCES}

${ }^{1}$ C. Kurtsiefer, S. Mayer, P. Zarda, and H. Weinfurter, Phys. Rev. Lett. 85, 290-293 (2000).

${ }^{2}$ L. Childress and R. Hanson, MRS Bull. 38, 134 (2013)

${ }^{3}$ W. F. Koehl, B. B. Buckley, F. J. Heremans, G. Calusine, and D. A. David, Nature 479, 84-87 (2011).

${ }^{4}$ D. A. Golter, T. Oo, M. Amezcua, K. A. Stewart, and H. Wang, Phys. Rev. Lett. 116, 143602 (2016).

${ }^{5}$ D. A. Golter, T. Oo, M. Amezcua, I. Lekavicius, K. A. Stewart, and H. Wang, Phys. Rev. X 6, 041060 (2016).

${ }^{6}$ S. J. Whiteley, G. Wolfowicz, C. P. Anderson, A. Bourassa, H. Ma, M. Ye, G. Koolstra, K. J. Satzinger, M. V. Holt, F. J. Heremans, A. N. Cleland, D. I. Schuster, G. Galli, and D. D. Awschalom, "Spin-phonon interactions in silicon carbide addressed by Gaussian acoustics," Nat. Photonics (published online).

${ }^{7}$ A. K. Geim and I. V. Grigorieva, Nature 499, 419 (2013).

${ }^{8}$ K. S. Novoselov, A. Mishchenko, A. Carvalho, and A. H. Castro Neto, Science 353, aac9439 (2016).

${ }^{9}$ T. T. Tran, K. Bray, M. J. Ford, M. Toth, and I. Aharonovich, Nat, Nanotechnol. 11, 37 (2016).

${ }^{10}$ T. T. Tran, C. Zachreson, A. M. Berhane, K. Bray, R. G. Sandstrom, L. H. Li, T. Taniguchi, K. Watanabe, I. Aharonovich, and M. Toth, Phys. Rev. Appl. 5, 034005 (2016).

${ }^{{ }^{11}}$ T. T. Tran, C. Elbadawi, D. Totonjian, C. J. Lobo, G. Grosso, H. Moon, D. R. Englund, M. J. Ford, I. Aharonovich, and M. Toth, ACS Nano 10, 7331 (2016).

${ }^{12}$ N. R. Jungwirth, B. Calderon, Y. Ji, M. G. Spencer, M. E. Flatté, and G. D. Fuchs, Nano Lett. 16, 6052 (2016).

${ }^{13}$ L. J. Martínez, T. Pelini, V. Waselowski, J. R. Maze, B. Gil, G. Cassabois, and V. Jacques, Phys. Rev. B 94, 121405(R) (2016).

${ }^{14}$ N. Chejanovsky, M. Rezai, F. Paolucci, Y. Kim, T. Rendler, W. Rouabeh, F. Fávaro de Oliveira, P. Herlinger, A. Denisenko, S. Yang, I. Gerhardt, A. Finkler, J. H. Smet, and J. Wratchup, Nano Lett. 16, 7037 (2016).

${ }^{15}$ Z. Shotan, H. Jayakumar, C. R. Considine, M. Mackoit, H. Fedder, J. Wratchup, A. Alkauskas, M. W. Doherty, V. M. Menon, and C. A. Meriles, ACS Photonics 3, 2490 (2016).

${ }^{16}$ B. Sontheimer, M. Braun, N. Nikolay, N. Sadzak, I. Aharonovich, and O. Benson, Phys. Rev. B 96, 121202(R) (2017).

${ }^{17}$ A. L. Exarhos, D. A. Hopper, R. R. Grote, A. Alkauskas, and L. C. Bassett, ACS Nano 11, 3328 (2017).

${ }^{18}$ M. Kianinia, S. A. Tawfik, B. Regan, T. T. Tran, M. J. Ford, I. Aharonovich, and M. Toth, ACS Photonics 4, 768 (2017).

${ }^{19}$ N. V. Proscia, Z. Shotan, H. Jayakumar, P. Reddy, C. Cohen, M. Dollar, A. Alkauskas, M. Doherty, C. A. Meriles, and V. M. Menon, Optica 5, 1128 (2018).

${ }^{20}$ A. L. Exarhos, D. A. Hopper, R. N. Patel, M. W. Doherty, and L. C. Bassett, Nat. Commun. 10, 222 (2019).

${ }^{21}$ M. Koperski, K. Nogajewski, and M. Potemski, Opt. Commun. 411, 158 (2018).

${ }^{22}$ R. Bourrellier, S. Meuret, A. Tararan, O. Stéphan, M. Kociak, L. H. G. Tizei, and A. Zobelli, Nano Lett. 16, 4317 (2016).

${ }^{23}$ G. Grosso, H. Moon, B. Lienhard, S. Ali, D. K. Efetov, M. M. Furchi, P. JarilloHerrero, M. J. Ford, I. Aharonovich, and D. Englund, Nat. Commun. 8, 705 (2017).

${ }^{24}$ Y. Xue, H. Wang, Q. Tan, J. Zhang, T. Yu, K. Ding, D. Jiang, X. Dou, J. Shi, and B. Sun, ACS Nano 12, 7127 (2018).
${ }^{25}$ G. Noh, D. Choi, J.-H. Kim, D.-G. Im, Y.-H. Kim, H. Seo, and J. Lee, Nano Lett. 18, 4710 (2018).

${ }^{26}$ A. Scavuzzo, S. Mangel, J.-H. Park, S. Lee, D. L. Duong, C. Strelow, A. Mews, M. Burghard, and K. Kern, Appl. Phys. Lett. 114, 062104 (2019).

${ }^{27}$ N. Mendelson, Z.-Q. Xu, T. T. Tran, M. Kianinia, J. Scott, C. Bradac, I. Aharonovich, and M. Toth, ACS Nano 13, 3132 (2019).

${ }^{28}$ N. Nikolay, N. Mendelson, N. Sadzak, F. Böhm, T. T. Tran, B. Sontheimer, I. Aharonovich, and O. Benson, Phys. Rev. Appl. 11, 041001 (2019).

${ }^{29}$ S. Nakhaie, M. Heilmann, T. Krause, M. Hanke, and J. M. J. Lopes, J. Appl. Phys. 125(11), 115301 (2019).

${ }^{30}$ A. Hernández-Mínguez, J. Lähnemann, S. Nakhaie, J. M. J. Lopes, and P. V. Santos, Phys. Rev. Appl. 10, 044031 (2018).

${ }^{31}$ F. Iikawa, A. Hernández-Mínguez, M. Ramsteiner, and P. V. Santos, Phys. Rev. B 93, 195212 (2016).

${ }^{32}$ C. Chakraborty, L. Kinnischtzke, K. M. Goodfellow, R. Beams, and A. N. Vamivakas, Nat. Nanotechnol. 10, 507 (2015).

${ }^{33}$ C. F. de las Casas, D. J. Christle, J. U. Hassan, T. Ohshima, N. T. Son, and D. A. David, Appl. Phys. Lett. 111, 262403 (2017).

${ }^{34}$ X. Li, G. D. Shepard, A. Cupo, N. Camporeale, K. Shayan, Y. Luo, V. Meunier, and S. Strauf, ACS Nano 11, 6652 (2017).

${ }^{35}$ W. P. Ambrose and W. E. Moerner, Nature 349, 225 (1991).

${ }^{36}$ O. Neitzke, A. Morfa, J. Wolters, A. W. Schell, G. Kewes, and O. Benson, Nano Lett. 15, 3024 (2015).

${ }^{37}$ J. Houel, A. V. Kuhlmann, L. Greuter, F. Xue, M. Poggio, B. D. Gerardot, P. A. Dalgarno, A. Badolato, P. M. Petroff, A. Ludwig, D. Reuter, A. D. Wieck, and R. J. Warburton, Phys. Rev. Lett. 108, 107401 (2012).

${ }^{38}$ J. R. Gell, M. B. Ward, R. J. Young, R. M. Stevenson, P. Atkinson, D. Anderson, G. A. C. Jones, D. A. Ritchie, and A. J. Shields, Appl. Phys. Lett. 93, 081115 (2008).

${ }^{39}$ I. Yeo, P.-L. de Assis, A. Gloppe, E. Dupont-Ferrier, P. Verlot, N. S. Malik, E. Dupuy, J. Claudon, J.-M. Gérard, A. Auffèves, G. Nogues, S. Seidelin, J.-P. Poizat, O. Arcizet, and M. Richard, Nat. Nanotechnol. 9, 106 (2014).

${ }^{40}$ J. Pustiowski, K. Müller, M. Bichler, G. Koblmüller, J. J. Finley, A. Wixforth, and H. J. Krenner, Appl. Phys. Lett. 106, 013107 (2015).

${ }^{41}$ S. Lazić, A. Hernández-Mínguez, and P. V. Santos, Semicond. Sci. Technol. 32, 084002 (2017).

${ }^{42}$ O. Arcizet, V. Jacques, A. Siria, P. Poncharal, P. Vincent, and S. Seidelin, Nat. Phys. 7, 879 (2011)

${ }^{43}$ E. D. S. Nysten, Y. H. Huo, H. Yu, G. F. Song, A. Rastelli, and H. J. Krenner, J. Phys. D: Appl. Phys. 50, 43LT01 (2017).

${ }^{44}$ S. Lazić, E. Chernysheva, A. Hernández-Mínguez, P. V. Santos, and H. P. van der Meulen, J. Phys. D: Appl. Phys. 51, 104001 (2018).

${ }^{45}$ H. Kumar, L. Dong, and V. B. Shenoy, Sci. Rep. 6, 21516 (2016).

${ }^{46}$ L. Rayleigh, Proc. London Math. Soc. s1-17, 4-11 (1885).

${ }^{47}$ S. Lazić, internal communication (2018).

${ }^{48}$ T. Müller, I. Aharonovich, L. Lombez, Y. Alaverdyan, A. N. Vamivakas, S. Castelletto, F. Jelezko, J. Wrachtrup, S. Prawer, and M. Atatüre, New J. Phys. 13, 075001 (2011).

${ }^{49}$ S. Völk, F. J. Schülein, F. Knall, D. Reuter, A. D. Wieck, T. A. Truong, H. Kim, P. M. Petroff, A. Wixforth, and H. J. Krenner, Nano Lett. 10, 3399 (2010).

${ }^{50}$ F. J. R. Schülein, K. Müller, M. Bichler, G. Koblmüller, J. J. Finley, A. Wixforth, and H. J. Krenner, Phys. Rev. B 88, 085307 (2013).

${ }^{51}$ M. Weiß, J. B. Kinzel, F. J. R. Schülein, M. Heigl, D. Rudolph, S. Morkötter, M. Döblinger, M. Bichler, G. Abstreiter, J. J. Finley, G. Koblmüller, A. Wixforth, and H. J. Krenner, Nano Lett. 14, 2256 (2014).

${ }^{52}$ A. Hernández-Mínguez, M. Möller, S. Breuer, C. Pfüller, C. Somaschini, S. Lazić, O. Brandt, A. García-Cristóbal, M. M. de Lima, Jr., A. Cantarero, L. Geelhaar, H. Riechert, and P. V. Santos, Nano Lett. 12, 252 (2012). 